\title{
Family Well-Being and Children with Epilepsy in Kilifi, Kenya: "When a Child Convulses his Family is Affected"
}

\author{
Nathaniel Kendall-Taylor ${ }^{*}, 1,2,3$, Caroline Kathomi $^{2}$, Kenneth Rimba ${ }^{2}$ and Charles R. Newton ${ }^{2,4}$ \\ ${ }^{I}$ Department of Anthropology, Los Angeles, University of California, USA \\ ${ }^{2}$ Centre for Geographical Medicine (Coast), Kenya Medical Research Institute, Kilifi, Kenya \\ ${ }^{3}$ FrameWorks Institute, Washington DC, USA \\ ${ }^{4}$ Clinical Research Unit, London School of Hygiene and Tropical Medicine, London, UK \\ ${ }^{5}$ Institute of Child Health, University College London, London, UK
}

\begin{abstract}
Background: The study was conducted with families of children with and without active epilepsy on the coast of Kenya, in Kilifi District. We established a context specific, ecocultural concept of "family well-being," and employed this concept to investigate how the presence of a child with persistent epilepsy impacts overall family functioning.

Methods: Dimensions of family well-being and the pathways through which epilepsy affects these dimensions were identified through quantitative and qualitative analysis of data from person-centered interviews and participant observation.

Results: Families in Kilifi constructed ideas of family well-being in terms of seven distinct dimensions: pleasure/pain experiences, social relationships, goals and values, daily routines, ties to land, resources, and physical health. Analysis revealed several aspects of well-being were compromised by caring for children with epilepsy, particularly family goals and values, daily routines, and social relationships. Examination of frequency counts of negative experiences in two subgroups of families of children with epilepsy (high and low frequency of symptoms) revealed that disease severity was associated with lower well-being and that some dimensions were more negatively affected than others.

Conclusions: Findings indicate that specific aspects of family well-being are affected by childhood epilepsy and suggest the need for improved seizure management and further research exploring associations of chronic childhood illness and family well-being. A better understanding of the relationship between family well-being and chronic pediatric illness can be used to develop more efficient family assessments and design more effective interventions targeted to specific family needs.
\end{abstract}

\section{INTRODUCTION}

Although research on the effects of chronic childhood conditions such as epilepsy have been concerned primarily with the child experiencing the condition, these conditions also have family consequences [1-11]. Family members may have to take time to provide care or devote significant resources in repeated attempts to obtain effective treatment for the child. Additionally, cultural perceptions of the illness may create family stigma, impacting the lives of all members. Family-focused research provides an ecocultural perspective on behavioral influences, illuminating the broad range of physical, social, cultural, and material pathways through which pediatric conditions shape the lives of children and the family members on whom they depend [12]

A family based approach is further justified by the importance of the family unit in both Western and nonWestern cultures [13-15], as well as by the negative impact of pediatric health conditions on the family unit [1-6]. In

*Address correspondence to this author at the FrameWorks Institute, $1776 \mathrm{I}$ Street NW, $9^{\text {th }}$ Floor, Washington, DC 20006, USA;

E-mail: nkendall-taylor@frameworksinstitute.org addition, because children's well-being is determined in large part by how they are cared for and their interactions within their families, a focus on the family provides an essential perspective on the effects of pediatric conditions on the children themselves [12]. Understanding how families are affected by pediatric conditions enables programs to address specific family needs, helps families to better manage these conditions, and may reveal strategies for maintaining well-being.

Past research, primarily in social psychology, has explored the effects of childhood epilepsy on families, and the impact of family factors on the child using surveys, psychological assessment measures, and one-time structured interviews $[1,6,16,17]$. An ethnographic approach provides an alternative perspective on the family consequences of chronic childhood epilepsy. By focusing on context and subjective experience, ethnographic methods facilitate a consideration of the wide range of family factors both affected by this condition and affecting its management. Furthermore, ethnography provides direct observational data on family behaviors, interactions, and naturally occurring conversations. In this way ethnography addresses some of the biases associated with participant report methods, most 
notably the differences between "reported" and "actual" behaviors [18].

We examine the utility of an anthropological concept of family well-being as a means to understand the effects of childhood epilepsy on the goals, values, activities and social relationships of caregivers. This concept stresses the culturally relative nature of wellness while acknowledging its universal characteristics [19]. We identify themes in local constructions of well-being in families living in Kilifi, a coastal district of Kenya. Using these themes, we analyze differences in the well-being of families of children with and without epilepsy and between two subgroups of families with children with different levels of epilepsy severity. We explore how specific aspects of well-being are affected by caring for children with epilepsy. We then employ frequency analysis to triangulate and verify these results $[20,21]$.

Themes of pleasure, social relationships, goals and values, routines, resources, and physical health have been recognized in previous ethnographic studies as separate components or dimensions of well-being [12, 22-26]. Drawing on qualitative data we show that families in Kilifi employ each of these dimensions in their conceptualizations of family well-being. We argue that these dimensions constitute a framework for conceptualizing family wellbeing that can guide researchers in observing the impact of a given health condition on family life and in designing interventions to address these impacts.

\section{METHODS}

\section{Study Area}

Kilifi town, the administrative center of the Kilifi District, lies roughly forty miles north of Mombasa, Kenya's second largest city, and forty miles south of the city of Malindi. The majority of people living in this coastal district reside in rural areas and rely on subsistence farming based on a biannual maize crop. Traditionally, Arab traders and the Islamic religion have had a strong presence on the coast. However since the time of British colonization, Christianity has become more prominent. The majority of the traditionally up-country tribes and some members of the coastal tribes practice various forms of Christianity [27]. Forty-seven percent of individuals in the study area identify as Christian, $13 \%$ Muslim, $24 \%$ Traditionalists, $12 \%$ as "other," and 4\% did not respond [28]. Some tribes, such as the Giriama, have managed to retain elements of their own unique religious beliefs and practices that form the basis of traditional healing and local epilepsy beliefs [27].

\section{Epilepsy in the Spirit System of Traditional Healing}

The local term for epilepsy in Kilifi District is Kifafa. In Mijikenda culture, illnesses, as well as social problems more generally, are caused by a system of spirits that can be divided into four categories: natural spirits (mapepo ya pori), Majini, ancestral spirits (mapepo ya kiasili), and Islamic spirits (mapepo ya kiislamu). Natural spirits are believed to cause a variety of diseases and conditions, including dizziness, and loss of appetite. Individuals may be infected by one of these spirits if they have passed the residence of a natural spirit, or if the spirit is attracted to the "sweetness" of the person's blood. Nyagu is the natural spirit most commonly considered to cause seizures in the traditional system. Seizures are believed to occur when the Nyagu comes to a child and to subside when the spirit leaves. Tremors and convulsions experienced during seizures are perceived as attempts to break free of the Nyagu. Nyagu is attracted by the smell or taste of certain children's blood. The spirit's preference for specific types of blood is used to explain why the spirit comes to some children and not others, as well as variation in the persistence of childhood seizures. If the Nyagu continues to visit the child, the spirit matures and reaches full maturity when the child is between the ages of five and ten, at which point the condition transforms from Nyagu to Kifafa.

Majini are evil spirits controlled by witches and are blamed for a host of problems ranging from insomnia to social and economic troubles. Witches are contracted by jealous parties to send Majini to specific individuals or families. Majini are sent in two ways. First, a witch can draw a special picture in the sand. Once the targeted individual walks over the drawing, the curse is cast. A witch may also send Majini directly to a person by verbally casting a spell. Seizures caused by witchcraft are considered easier to treat than the Nyagu type because the Majini are not as strong or persistent as Nyagu.

Ancestral spirits are the descendants of original Mijikenda ancestors. The Mijikenda believe that their first traditional healer was Mepoho, the mother of two sisters, Matsezi and Mbodze. These daughters were also powerful healers and had 12 children of their own who became renowned healers. The spirits of these 12 children are the primary Mijikenda ancestral spirits, and can cause various problems from bad luck in farming to headaches and infertility. Other secondary ancestral spirits may also cause these problems. Anyone who has died has the possibility of becoming a spirit of this type and affecting the world of the living. Ancestral spirits are believed to cause problems because of unfulfilled desires. Seizures may be transferred between family members if specific rituals are not performed following the death of a member with epilepsy. If these rituals are not performed, the spirit of the dead relative may return and the next child born may have the condition.

Islamic spirits are the spirits of important Muslim teachers and religions figures and are believed to be more powerful than their Mijikenda counterparts. However, these spirits are not believed to cause seizures.

\section{Biomedical Treatment in Kilifi}

Biomedical treatment in Kilifi is provided by a government hospital (Kilifi District Hospital), government health centers, and private clinics. Kilifi District Hospital $(\mathrm{KDH})$, the only hospital in Kilifi District, is located in Kilifi Town. Thirty-four government health centers and dispensaries are dispersed throughout the district, staffed by doctors, nurses, and health workers with various degrees of training in biomedicine. In addition, approximately 25 private clinics operate in the district, which vary widely in staff training and medication supplies.

$\mathrm{KDH}$, in conjunction with Kenya Medical Research Institute-Coast, operates a special clinical program for children with epilepsy. It provides free assessment and clinical services and anti-epileptic drugs (AEDs) at subsidized prices and is staffed by three clinicians with 
experience in the management of epilepsy. Government health centers do not offer this level of service. The latter facilities are permitted to prescribe only the AED phenobarbitone, and lack supplies of this medication. The staff members at these health facilities and at private clinics have little or no training in epilepsy diagnosis and management.

\section{Childhood Epilepsy: Definition and Perceptions of Families in Kilifi}

Epilepsy, or seizure disorder, is a condition characterized by recurrent (two or more) epileptic seizures, unprovoked by an immediate identified causes $[29,30]$. The case definition of epilepsy is based on a patient's clinical history and the presence and pattern of physical symptoms. Epilepsy can be the result of many neurological conditions including brain trauma, genetic abnormalities, and central nervous system infections.

The epilepsy rate in Kilifi, where the present study was conducted, is estimated to be nine percent--one of the highest on the African continent [31,32]. The high prevalence of epilepsy in the developing world may be related to factors occurring more frequently in developing countries, such as more prevalent alcoholism and childhood trauma [33], as well as poor sanitation including parasitic contamination [34]. Other research suggests that the high rates of epilepsy in developing countries may reflect marriage practices in which individuals with epilepsy are only suitable partners for other individuals with the disorder, or may result from perinatal head trauma associated with the birthing practices used in some cultures [35]. Striking gaps in Kilifi between the prevalence of epilepsy and the number of individuals receiving proper biomedical treatment make these statistics even more dramatic [36]. Previous attempts to bridge this treatment gap have been largely unsuccessful. In deciding where to seek treatment for their child, families of children with epilepsy face a complicated treatment-seeking process. Initially they must decide whether to go for traditional or biomedical treatments and subsequently whether to adhere to one, both, or neither of these treatments. Research in Kilifi suggests that the local system of beliefs regarding illness causation and the process by which families evaluate treatment outcomes are fundamental influences on these decisions [18].

\section{Sample}

The sample of families with children with epilepsy was selected from a larger cohort identified in a previous epidemiological study [37]. For the present study, 111 children were selected that met the selection criteria of having active epilepsy and being between six and eleven years of age. The final sample for the current study comprised the children of 107 families that consented to participation and completed an initial survey providing data on family demographics, the child's epilepsy, and aspects of treatment choice. Due to the time and resources required to conduct and analyze long-term family-centered ethnography, results of the survey were used to select a smaller sub-group of the sample for in-depth qualitative research.

In the survey, family members indicated, among other things, how frequently their child experienced seizures.
Based on reports of seizure frequency, the sample was divided into two sub-groups representing children with "severe epilepsy," with frequent seizures, versus more "moderate epilepsy," with less frequent seizures. Seizure frequency was used as the measure of severity because of its suspected association with family burden and quality of life issues [38]. Epilepsy treatment history was also gathered in the survey. However, due to lack of data on actual rather than reported adherence to drug treatments, we were not able to determine the intractability of seizures for children in the sample [39]. In addition, the rural, home-based context of survey administration and the distance of most families from the district hospital, made assessment of drug adherence difficult.

Severe and Moderate Epilepsy Groups were formed by randomly selecting four families of children at each severity level. Despite differences in severity, the children in both epilepsy (or index) groups experienced persistent seizures and had been diagnosed as having "active" persistent epilepsy [37]. One "control" family was recruited for each of the eight index families. Control families were selected based on the presence of a child without epilepsy of approximately the same age as the corresponding index child. Each control family was also individually matched to the index family based on ethnicity, religion, location, resources, and family size.

With the help of a local Giriama research assistant, the primary researcher followed these 16 families for nine months and gathered data through interviews and participant observation, an anthropological method in which the researcher(s) participates in daily activities, talks informally with neighbors, observes how the families goes about their daily lives, and takes careful field notes on interactions and events. During field visits, the researchers spent the day at the families' homesteads and developed close relationships with the families as well as with other members of the community.

The two researchers also interviewed family members in their native language using the person-centered interview methodology [40]. Interviews focused on topics such as goals, ambitions, experiences, and subjects such as daily routines. The transcripts of these interviews were the primary data in comparative and frequency analysis. Data from informal discussions with family members and members of their communities, as well as field observations, were used to confirm and support findings from comparative analysis of interview transcripts.

Qualitative data were reviewed using a grounded theory approach [20, 41, 42]. Using this technique, analysis generated seven themes that contribute to the well-being of families in Kilifi. Working with this set of themes, transcripts and field notes were coded. The themes were modified and refined during coding to accommodate cases that did not fit the system of emerging themes [20]. Comparisons between index and control groups and between epilepsy severity sub-groups were then made to identify similarities and differences in how the groups discussed different dimensions of well-being [43, p. 84-95].

Using a method of quantitative content analysis $[20,44-$ 46], transcripts were coded for statements pertaining to 
threats or barriers to well-being, referred to here as negative utterances. These utterances were then tallied for each of the seven themes of well-being. The frequencies were compared for the index versus control families and for index families in the Severe versus Moderate Epilepsy Groups. The frequencies of negative utterances in each well-being category/theme were compared using standardization counts to control for different numbers of total utterances [21]. A focus on negative utterances was justified by an interest in adverse family consequences of caring for a child with epilepsy. Standardizing counts improved between-group comparisons by adjusting for differences in data volume; and facilitated interpretation of the group comparisons on each dimension of well-being by adjusting for differences in the total number of counts per dimension.

\section{RESULTS}

\section{The Well-Being of Families with and without Children with Epilepsy}

Analysis of qualitative data from interviews, informal discussions and observations from the 16 families in the study yielded evidence for the following seven themes associated with conceptions of family well-being: goals and values, daily routines, social relationships, resources, physical health, pleasure/pain experiences, and ties to land. These themes comprise a context-specific understanding of well-being for a select group of families in Kilifi, Kenya, half of which were caring for a child with epilepsy. Nevertheless, the themes are similar to those that have emerged in previous studies of well-being in other regions of the world, suggesting components of well-being that are common across conditions and cultures [12, 22-26]. The individual themes are not exclusive categories as families frequently discussed problems or circumstances that were exemplars of multiple themes. However, the set of themes accounted for all aspects of family well-being found in this project's ethnographic data. Findings regarding these themes and comparisons between index and control families and between index families divided according to the severity of the child's epilepsys (Severe vs Moderate) are presented below and summarized in Tables $\mathbf{1}$ and $\mathbf{2}$.

\section{Goals and Values}

Individuals assign cultural significance to and derive meaning from specific life events, activities, and objects. These culturally structured meanings create a system of goals and values that individuals strive to achieve and maintain. Therefore, goals and values are important in individual and group conceptualizations of the "good life" $[25,47]$. The significance and meaning assigned to particular events, activities, and objects vary across cultures and structure goals, values, and standards that individuals use in assessing experiences [47, 48].

Achieving, maintaining, and striving towards goals and values contributed to members' ideas of family well-being in Kilifi. Goals and values were the most frequently discussed of the seven themes (see Table 3). Family members who were able to achieve goals or who saw goals as attainable spoke positively of their well-being. Family members who persistently failed to

Table 1. Summary of Index-Control Family Comparison

\begin{tabular}{|l|l|l|}
\hline & \multicolumn{1}{|c|}{ Index Families } & Control Families \\
\hline \hline Goals and Values & Unattainable & Attainable \\
\hline Daily Routines & Unpredictable, disrupted & Predictable, established \\
\hline Social Relationships & Family and individual stigma, strained intra family relations & Generally positive intra and extra family relations \\
\hline Resources & Epilepsy treatment as a pressure on family resources & General resource shortages \\
\hline Physical health & Poor family health due to presence of epilepsy & Generally positive \\
\hline Pleasure/Pain Experiences & Physical fatigue/pain from hard labor, pain from stress/anxiety & Physical fatigue/pain from hard labor \\
\hline Ties to Land & No marked difference between index and control groups & \\
\hline
\end{tabular}

Table 2. Summary of Severe Epilepsy Group - Moderate Epilepsy Group Comparison

\begin{tabular}{|l|l|l|}
\hline & \multicolumn{1}{|c|}{ Severe Epilepsy Group } & Moderate Epilepsy Group \\
\hline \hline Goals and Values & Seriously affected, unattainable & Affected but possibly attainable in the future \\
\hline Daily Routines & Seriously disrupted & Infrequently disrupted, relatively predictable \\
\hline Social Relationships & $\begin{array}{l}\text { Family stigma, individual stigma, tension between } \\
\text { members }\end{array}$ & $\begin{array}{l}\text { Lack of family stigma, infrequent individual stigma, infrequent } \\
\text { intra family tension }\end{array}$ \\
\hline Resources & Frequent seizures as a serious drain on resources & General concerns, similar to those of control families \\
\hline Physical health & Very poor & Affected but not very poor \\
\hline Pleasure/Pain Experiences & $\begin{array}{l}\text { Pronounced anxiety over child's condition causing } \\
\text { physical pain }\end{array}$ & $\begin{array}{l}\text { Less pervasive anxiety and limited discussion of pain from } \\
\text { anxiety }\end{array}$ \\
\hline Ties to Land & No marked difference between groups & \\
\hline
\end{tabular}


achieve cultural goals or uphold values or who saw goals or values as unattainable had negative views of their family's wellbeing.

Family autonomy and self-sufficiency were values discussed by all families. One family member explained, “...the ability of the family to get those things they need on their own...not asking everyday for these small things." Each family member's economic contribution was also a frequently discussed goal. The self-sufficiency and independence of children were other important values. One father said, "My goal is that each of my children will take care of themselves." Another value was the family's ability to work together, cooperate, and get along as a unit. As one family member explained, "A family that is good is the one that is able to cooperate... This family will be happy and the one that is not able will ruin itself." Family members also shared the goal of having their children attend school, obtain salaried jobs, and return to the family to support other members. Ownership was another frequently mentioned goal. Family members expressed a desire to increase or begin to develop livestock holdings. Building a "permanent" house was a common goal. Most families in the sample lived in mud huts, which had to be frequently repaired and re-built. A permanent house solidified a family's reputation in the community and was a tangible symbol of a family's success.

Index vs control groups. Most of the members of control families believed key cultural goals were attainable and were positive and optimistic in their ability to uphold values. In contrast, the members of index families frequently perceived their child's condition as a barrier to attaining goals. Many of these family members reported that the condition impeded the child from going to school, becoming independent, and contributing to the family. Four of the eight index children did not go to school. In each of these cases siblings either were in school or had attended school in the past. Family members attributed the index child's absence from school to epilepsy and felt that the lack of schooling would prevent the child from getting a job, being successful, or supporting parents in old age. The following vignette illustrates these perceptions:

Interviewer: What effect do diseases have on your family? For example if Ndugu did not have epilepsy would there be a difference?

Father: There would be a difference because he would be playing with other children, going to school, herding cattle over the weekend, but now he can't do anything...not even for himself...

Mother: [if he did not have epilepsy] he would be happy and would be able to go everywhere like school. Now he cannot go to school. He is just here at home...he will never have a job...he will never bring money..."

Index family members also felt that, because of the chronic nature of epilepsy, the child would require ongoing care.

Another aspect of family well-being that was affected by caring for a child with epilepsy was the family's ability to make financial plans and to achieve material goals such as acquiring livestock or building a permanent house. All families in the sample sought some type of treatment for their child's condition during the course of the study. Families reported that the unpredictability of periods of especially frequent seizures and associated treatment needs hindered their ability to save money. One father explained, "You must never spend all the money...You must always stay with some because this illness comes suddenly."

Severe vs Moderate Epilepsy Groups. Severe Epilepsy Group families were less optimistic about their ability to attain cultural goals than Moderate Epilepsy Group families. Only one index child in the Severe Epilepsy Group attended school and the child's attendance was infrequent and sporadic. In contrast, three of the four index children in the Moderate Epilepsy Group went to school. The fourth Moderate Epilepsy Group index child was not attending school at the beginning of the project due to increased seizure frequency but had returned to school by the end of the study. The two groups also differed in their perceptions of the index child's ability to become independent. Members of all Severe Epilepsy Group families reported little hope of the index child ever being independent. One family in the Moderate Epilepsy Group reported similar views, but the remaining three Moderate Epilepsy Group families were more optimistic and thought it was possible that their children would one day be able to live on their own.

\section{Daily Routines}

Everyday activities are, to a large degree, responsible for shaping the lives, experiences, and "developmental pathways" of parents, children and families [12]. Weisner argues that examining the quality and predictability of daily routines facilitates a context-specific ecocultural picture of family well-being. In accord with Weisner's work, family members in Kilifi discussed the importance of a predictable and productive daily routine in family well-being.

The two strategies that shaped the quality and consistency of informants' daily lives were cooperation and coordination. The maintenance of these strategies was viewed as vital to family functioning on a day-to-day basis and as determinants of a family's more general level of wellbeing. In an informal discussion, one family member said, "A family that is able to do things together... and with people who work hard by themselves... will be able to get their work done...they will be happy." Families that lacked cooperation and coordination in their daily lives were seen to lead chaotic lives and were depicted as un-well. Family members defined cooperation as two or more members working together on the same task. They defined coordination as dividing a task into constituent components and allocating components to individual family members. Striking a balance between cooperation and coordination benefited family well-being, while an imbalance of the two strategies or the absence of either resulted in compromised family well-being.

Index $v s$ control groups. While events occasionally caused control families to deviate from their daily routines, these events were viewed as neither highly disruptive nor atypical and included occurrences such as a fever requiring a parent to take the child to hospital, anniversary celebrations, or weddings. In contrast, the members of families with a child with epilepsy described children's seizures as so unpredictable and disruptive to family routines that they compromised the quality of family life. In five of the eight index families, a family member stayed at home with the child at all times. The productivity of the members of these five families was reduced and they were often prevented 
from work that required them to leave the home, such as farming or casual labor. The index children were also unable to participate in many necessary daily tasks, including traveling to obtain water, foraging for firewood, cooking, and farming. A mother explained, "Others go to do these chores and she stays to sweep... She cannot do much work because... she will fit." Index family members frequently had to abandon their normal daily tasks and routines to seek help for the child.

Severe vs Moderate Epilepsy Groups. Seizures and treatment seeking frequently disrupted the daily routines of families in the Severe Epilepsy Group. A mother of a child with infrequent seizures explained, "I can leave her and she will be fine but others who fit every hour can not be left alone..." While the daily routines of four Moderate Epilepsy Group families were occasionally disrupted by seizures, their lives more closely approximately those of control families.

\section{Social Relationships}

Past research suggests that the quality of social relationships is an important component of well-being (25, 49; Izquierdo, 2005 \#150). Interviews, observations, and informant conversations confirmed the relevance of relationships between members of a family and with others in the community. Social interactions with neighbors and members of the community were an important source of emotional and financial support for families. Family members who were able to seek advice from neighbors and whose neighbors came to them for support derived satisfaction from these interactions and emphasized the importance of amicable relationships for their family's wellbeing. One father said, "We cooperate...we share. If I need advice I go there and ask...We do more than just greet, we are together here." Relationships with neighbors could also be a source of apprehension and fear, and threaten a family's well-being. Family members recounted incidents of community jealousy towards successful families. In Kilifi, jealousy is a perceived source of danger. Jealousy and envy are believed to precipitate witchcraft and evil curses, causing various misfortunes including economic hardship, illness, and even death.

The ability of family members to get along with each other was another dimension of social relationships impacting family well-being. One family member reported, "A family must cooperate and agree with each other. One [a family] that fights all the time will always fail...They will not be able to get food and will be sad if they are not able to get along." Families whose members talked to one another each night about daily events were depicted as good families. Other key abilities and determinants of positive family interaction included the ability to solve problems as a family and to make daily and long-term plans.

Index vs control groups. Social tensions were present in both index and control families. Two control families were involved in ongoing land disputes that strained their relationships with neighbors. Fieldwork revealed that these types of disputes are relatively common in Kilifi, where property is of vital symbolic and material importance and land boundaries are often ambiguous. Intra-family tension associated with severe alcohol abuse was observed within another control family. This was also a relatively common problem affecting families in Kilifi. Control family data on intra and extra family interactions were otherwise positive.

In index families, the child's epilepsy imposed an additional strain on intra and extra family social relationships. Specifically, three index families reported stigmatization of the family that they attributed to their children's epilepsy. Observations of relationships between these families and their wider community, as well as conversations with other members of their community, confirmed this perception. In each case, stigma strained relationships with other community members and isolated the family. Members of these three families felt that being stigmatized impacted their family's well-being. In two of these three families, more than one family member had seizures, a circumstance treated by the community in Kilifi as a sign of a family's involvement in witchcraft. A member of one stigmatized family explained, "They [the neighbors] think that he has epilepsy because they think we are witches." In addition to the family-level stigma, members of both index and control families reported that their communities believed persons with epilepsy were "crazy" and that they had "ruined" brains and were unable to participate in normal activities. Negative intra family interactions resulting from the child's epilepsy were observed in five of the eight index families. Sibling resentment of the index child was reported and attributed to unequal distribution of family time and financial resources because of the child's condition. One parent explained, "...they [the child's siblings] become angry sometimes...They see that we spend time getting medicine for that one's epilepsy...we have spent a lot of money... They think this is not fair to them and they get mad at him." In addition, family interactions were occasionally strained by having to deal with frequent seizures. Family members, especially siblings, reported feeling burdened and on occasion were observably irritated about having to stay home and take care of the index child.

Severe $v s$ Moderate Epilepsy Groups. Higher seizure frequency was associated with increased family stigmatization. The three families that were stigmatized by their neighbors and communities were all in the Severe Epilepsy Group. Moreover, family members reported frequent occurrence of stigmatization for all index children in the Severe Epilepsy Group but for only one index child in the Moderate Epilepsy Group. Instances of stigmatization for the other three index children in the Moderate Epilepsy Group were infrequent. Compared with the index children in the Moderate Epilepsy Group, those in the Severe Epilepsy Group were also perceived as having more "seriously" damaged brains and as being less competent.

The quality of intra family interactions was also related to seizure frequency. Family members in the Severe Epilepsy Group reported that the child's siblings felt animosity towards the index child. The reason given for this animosity was perception that the index child was receiving more than their fair share of the family's resources. Sibling resentment was not observed in Moderate Epilepsy Group families. Observations additionally revealed instances in which the activities of each Severe Epilepsy Group family were disrupted by the index child or in which members had disputes regarding responsibility for the index child's care. 
Similar episodes were observed in only one Moderate Epilepsy Group family.

\section{Resources}

Past research documents the importance of access to resources, the absence of which Edgerton refers to as "environmental stress," as critical to family well-being [50]. Families in the sample varied significantly in their access to resources. Several families had large herds of cattle, owned large properties with many palm trees, and had coral quarries on their land. Other families had no cattle, lived on others' land or on property without significant resources, or were unable to secure casual employment. Most families did not have regular incomes or ongoing access to financial resources, and lacked disposable income and financial savings. To obtain cash to purchase food staples and other daily needs, families sold surplus crops, coconuts, palm wine, livestock, engaged in intermittent casual labor or sought loans from neighbors.

Family members reported that one way they assessed their family well-being was by comparing their resources to those of other families. Family members also assessed wellbeing in relation to the resources of a "typical" family. One mother explained, "It is normal that a family has cattle, a nice house and a large shamba... we don't have these things."

Limited access to resources also exerted a negative influence on other aspects of family well-being. Members of families lacking resources reported strained community relationships because they were perceived as "poor" and were frequently forced to seek loans from other community members. A lack of resources also put pressure on a family's goals by limiting access to education for children and casting doubt on the attainability of material goals. Daily routines were also strained in these families as a result of the need for family members to seek unpredictable daily labor in the fields of others; and physical health was affected by limiting the family's ability to feed its members or by reducing the family's ability to obtain health care.

Index vs control groups. Resource shortages were a common topic of discussion for both groups of families. Because of matching procedures, index and control families faced similar resource challenges. However, the presence of a child with epilepsy imposed an additional strain on resources. Control families expressed concerns about lack of money, living in substandard housing, and poverty relative to other families in their community. While families with a child with epilepsy had similar concerns, their discussion focused on the resource strains resulting from the child's condition. Index families had to pay for multiple treatments for the index child and associated transportation costs. Because of the chronic nature of epilepsy, and the failure of both biomedical and traditional treatments to fully control seizures, treatment-seeking was an on-going process and placed continual demands on financial resources. Index family members also emphasized that seizure unpredictability and treatment needs made it difficult to make long-term financial plans. Five of the eight index families had reduced availability of labor resources because caregivers had to remain at home with the index child; and in six index families, the children did not participate in normal household activities performed by siblings of similar age. As is the norm in Kilifi, all control children helped with household tasks.

Severe vs Moderate Epilepsy Groups. Families in the Severe Epilepsy Group expressed concerns about the drain on family resources imposed by frequent treatment visits. These families recognized the need for treatment and traveled considerable distances to receive it from either biomedical clinics or traditional healers. Families in the Moderate Epilepsy Group did not take their children as frequently for treatment. Two Moderate Epilepsy Group families traveled to the District Hospital about once every three months to receive anti-seizure medications. The remaining two Moderate Epilepsy Group families sought treatment only on those infrequent occasions when the child experienced a spike in seizure frequency. Treatment seeking thus imposed a less frequent strain on family resources in the Moderate Epilepsy Group than in the Severe Epilepsy Group.

\section{Physical Health}

Physical health is perhaps the most obvious aspect of well-being [23, 26, 51, 52]. In commenting on physical health, family members in the sample emphasized the importance of eating well and being free of major disease. The ability to drink tea with milk every morning and eat meat at least once a day were concrete standards of family health and well-being. In answering a question about his daughter's health, one father replied, "Healthy? Just look at her, she is big and fat and eats meat every day...You can see that she is good!" Another father stated, "We do not have strength/health. We eat sima (cornmeal porridge) and mboga (vegetables) every day...Look how thin we are." In describing the value of being free from major disease or chronic illness, family members viewed some ill health, including the occasional malarial flu, as "normal." A mother in one of the control families explained, "We do not have any big problems of health, we have good health...Right now we have fever but this is normal, it will go."

Index vs control groups. Physical health was a frequent topic in both groups of families. Control families viewed child health problems as relatively common occurrences that did not impinge on the family's health. In contrast, the members of all families with a child with epilepsy perceived the child's seizures as having compromised the health of the family unit. Index families also had pessimistic views about the prospects of improvements in family health.

Severe vs Moderate Epilepsy Groups. Severe Epilepsy Group families had more negative perceptions of family health than Moderate Epilepsy Group families. One father in the Severe Epilepsy Group asked, "If you had this child in your family would you feel your family had health? ...this child lowers our health... until it [epilepsy] is healed, we will have bad health." Members of Moderate Epilepsy Group families also perceived the child's condition as affecting their family health, but thought that they could still have a healthy family despite the condition.

\section{Pleasure/Pain Experiences}

Clark argues that pleasure is a universal dimension of well-being but also claims that the way pleasure and pain are experienced and their meanings are in large part culturally 
determined [24]. Consistent with this view, family members in the Kilifi sample often spoke about their well-being in terms of experiences of pleasure and pain. Most discussion of pleasure or pain focused on the sensory experiences of manual labor, rest, and the presence or absence of physical ailments. Working on a shamba (farm) to earn money is the cornerstone of daily life in Kilifi. Families utilized time- and labor-intensive methods of agriculture, which necessitated long hard days of work. Complaints about being tired, sore, or without happiness "in the body" were frequent subjects of discussion within families and with visitors. Physical illnesses believed to result from excessive labor were also frequently discussed. While individuals had to work hard throughout the day, most also had time to rest with family in the evening. Family members described these periods of rest as a primary source of physical pleasure and a vital aspect of their well-being.

Members of one control family reported, "... our bodies have much happiness." They attributed this pleasure to their financial ability to hire labor and avoid excessive physical exertion. As the owners of a successful general store, this family had the financial means to avoid daily manual labor, and saw this capacity as a source of physical pleasure. Members of another family that was poor by local standards and had no land of its own were forced to work in the farms of neighbors for daily wages to buy food staples. Members reported feeling constant fatigue and physical pain because of their long hard hours working in the fields and their lack of "time to rest."

Index vs control groups. Although the index and control families both complained about bodily discomfort associated with fatigue and physical labor, only index family members described physical discomfort related to anxiety. They attributed anxiety to worries regarding the child with epilepsy and effectiveness of the child's treatment. One father of an index family said, "This is not something that you want for your first son. It has brought me anxiety... and my body hurts." A parent of another index family said, "Kifafa [epilepsy] is different...because you have to keep doing this [treatment] and you are never able to stop worrying...you are never able to rest from this worry...you feel this worry very much." Members of each index family described frustration from on-going attempts to find more effective treatments and reported anxiety and physical discomfort when the child experienced seizures.

Severe vs Moderate Epilepsy Groups. Comparison of the epilepsy severity groups revealed the same pattern. Severe Epilepsy Group families appeared highly stressed and frequently discussed anxiety and its manifestation as physical pain. While families in the Moderate Epilepsy Group discussed anxiety associated with the child's condition and treatment, descriptions of anxiety causing physical pain were less common. Moderate Epilepsy Group families also expressed less anxiety about their child's condition than those in the Severe Epilepsy Group.

\section{Ties to Land}

Previous studies have found that the physical environment and changes to this environment contribute to family well-being $[22,26,49,53,54]$. This theme was echoed by the emphasis family members placed on land ownership and cultivation as a source of feelings of continuity. One man, who had lived on the same piece of land throughout his entire life explained, "I am completely used to it here...It is mine and when I am here I am happy...I would be lost without this land." Lack of land ownership was viewed as having a negative effect on family well-being. Members of one family had squatted on the same piece of land for over fifty years. With notable irony they discussed owning the trees they had planted, but not the land on which the trees were planted. In this family, lack of ownership was an emotional subject. The grandmother said, "We own the trees because I have planted them many years ago with my husband who died...but the land...it is owned by someone else because my husband was sick when the time came to make our claim and could not go."

Comparisons of index and control groups and Sever and Moderate Epilepsy Groups did not reveal any identifiable differences with respect to the theme of ties to land.

\section{Frequency Analysis}

Table 3 presents the results of frequency analysis of control and index family data. Columns 1 and 3 provide the total number of utterances related to each dimension of wellbeing for index and control groups respectively. Columns 2 and 4 list the number of utterances coded as negative for index and control families, respectively. Column 5 provides the standardized negative counts for the control group. These standardized negative counts were computed by dividing the total number of index utterances for each dimension by the total number of control utterances related to that same dimension. This ratio was multiplied by the raw count of control group negative utterances for the dimension to obtain standardized counts. Control group counts were standardized to account for the differences in total utterances between index and control groups and enabled index-control group comparison of negative utterances for each dimension. Column 6 provides the differences between the index group's negative utterances and the control group's standardized negative utterances. Column 7 provides a standardized measure of these differences and allows for comparison across the seven dimensions. Differences were standardized to account for variations between the dimensions in total utterances. Comparing standardized differences between dimensions reveals the relative effect of the child's epilepsy on each aspect of well-being.

Consistent with the findings of the comparisons presented in the previous section, positive values in six of the seven cells in column 6 indicate that index family counts of negative utterances were higher than standardized counts in the control group for all dimensions except "ties to land." Relatively large values in column 7 for the dimensions of daily routines (215) and goals and values (203.51) suggest that these aspects of well-being were more affected by caring for children with epilepsy than the other aspects. The standardized difference was also large in the social relationship theme (191.97), indicating that this dimension of well-being was also strongly affected by caring for these children. In 11 of the 14 well-being theme counts (seven themes for both index and control groups) no one family's count fell more than two standard deviations outside of the group mean. The remaining three counts were within 2.5 
standard deviations of the mean. This suggests that the counts were representative of all families and were not driven by outlier cases [55].

Table 4 presents the results of frequency analysis of the Severe and Moderate Epilepsy Groups. Columns 1 and 4 provide the total number of utterances for each dimension of well-being for Severe and Moderate Groups, respectively. For each group, respectively, columns 2 and 5 indicate the number of total utterances, and columns 3 and 6 provide standardized counts of negative utterances. Because of the differences in total utterances, negative utterance counts were standardized to allow for between-group comparisons. Column 7 presents the differences between the standardized negative counts for each dimension. Positive values in column 7 indicate that family perceptions and experiences for six of the seven dimensions were more frequently negative for families in the Severe Epilepsy Group than in the Moderate Epilepsy Group, as is consistent with results of comparisons presented in the previous section.

Comparison of values in column 8 reveals the themes that were relatively more compromised in Severe Epilepsy Group families. The large values for the dimensions of goals and values (63.99) and resources (42.58) indicate that Severe
Epilepsy Group families viewed these aspects of well being as particularly problematic. The differences in standardized counts and standardized difference values are less dramatic for Severe versus Moderate Group comparisons (columns 7 and 8 of Table 4) than for index versus control comparisons (columns 6 and 7 of Table 3), suggesting that the presence of uncontrolled seizures is a more critical determinant of family well-being than seizure severity.

\section{DISCUSSION}

Analysis of ethnographic data from person-centered interviews, informal discussions, and participant observation with families in Kilifi identified seven dimensions of family well-being: pleasure/pain experiences, social relationships, goals and values, daily routines, ties to land, resources, and physical health. These results are consistent with dimensions of well-being separately identified in past studies in other cultural contexts, suggesting that these may be important cross-cultural determinants of family well-being. In support of the cross-cultural nature of these dimensions, the present study found evidence for each component of well-being in all participating families.

Table 3. Index-Control Family Frequency Analysis

\begin{tabular}{|l|c|c|c|c|c|c|c|}
\hline & Index Families & \multicolumn{4}{|c|}{ Control Families } & \multicolumn{2}{c|}{ Comparison } \\
\cline { 2 - 8 } & $\mathbf{( 1 )}$ & $\mathbf{( 2 )}$ & $\mathbf{( 3 )}$ & $\mathbf{( 4 )}$ & $\mathbf{( 5 )}$ & $\mathbf{( 6 )}$ & $\mathbf{( 7 )}$ \\
\cline { 2 - 8 } & Total Utterances & $\begin{array}{c}\text { Negative } \\
\text { Utterances }\end{array}$ & $\begin{array}{c}\text { Total } \\
\text { Utterances }\end{array}$ & $\begin{array}{c}\text { Negative } \\
\text { Utterances }\end{array}$ & $\begin{array}{c}\text { Stand. } \\
\text { Negative }\end{array}$ & $\begin{array}{c}\text { Differences } \\
\text { in Counts }\end{array}$ & $\begin{array}{c}\text { Stand. Differences } \\
\text { in Counts }\end{array}$ \\
\hline \hline Goals and Values & 443 & $\mathbf{2 5 4}$ & 272 & 31 & $\mathbf{5 0 . 4 9}$ & 203.51 & 203.51 \\
\hline Daily Routines & 238 & $\mathbf{1 2 6}$ & 120 & 9 & $\mathbf{1 7 . 8 5}$ & 108.1 & 215 \\
\hline Social Relationships & 255 & $\mathbf{1 3 8}$ & 216 & 48 & $\mathbf{5 6 . 6 7}$ & 81.33 & 191.97 \\
\hline Physical Health & 212 & $\mathbf{1 6 4}$ & 95 & 57 & $\mathbf{1 2 7 . 2}$ & 36.8 & 85.71 \\
\hline Pleasure/Pain & 142 & $\mathbf{1 2 5}$ & 69 & 44 & $\mathbf{9 0 . 5 5}$ & 34.45 & 132.43 \\
\hline Ties to Land & 95 & $\mathbf{5}$ & 43 & 4 & $\mathbf{8 . 8 4}$ & -3.84 & -27.73 \\
\hline
\end{tabular}

Standardized negative $=$ Total index family utterances/ Total control family utterances*control family negative utterances. Standardized difference in counts=(index family total goals and values+control family total goals and values)/(index family total theme $\mathrm{X}+$ control family total theme $\mathrm{X}$ )* Difference in counts.

Table 4. Index-Control Family Frequency Analysis

\begin{tabular}{|c|c|c|c|c|c|c|c|c|}
\hline & \multicolumn{3}{|c|}{ Severe Epilepsy Group } & \multicolumn{4}{|c|}{ Moderate Epilepsy Group } & \multirow{2}{*}{$\begin{array}{c}\text { Comparison } \\
(8)\end{array}$} \\
\hline & (1) & (2) & (3) & (4) & (5) & (6) & (7) & \\
\hline & Total & $\begin{array}{c}\text { Total } \\
\text { Negative }\end{array}$ & $\begin{array}{l}\text { Stand. } \\
\text { Negative }\end{array}$ & Total & $\begin{array}{c}\text { Total } \\
\text { Negative }\end{array}$ & $\begin{array}{l}\text { Stand. } \\
\text { Negative }\end{array}$ & $\begin{array}{l}\text { Differences in } \\
\text { Counts }\end{array}$ & $\begin{array}{l}\text { Stand. Differences } \\
\text { in Counts }\end{array}$ \\
\hline Goals and Values & 211 & 134 & 140.99 & 222 & 77 & 77 & 63.99 & 63.99 \\
\hline Daily Routines & 85 & 49 & 88.2 & 153 & 77 & 77 & 11.2 & 20.85 \\
\hline Social Relationships & 164 & 91 & 91 & 93 & 45 & 81.1 & 9.9 & 17.2 \\
\hline Resources & 196 & 142 & 142 & 106 & 61 & 112.97 & 29.03 & 42.58 \\
\hline Physical Health & 124 & 101 & 101 & 88 & 63 & 88.77 & 12.23 & 25.56 \\
\hline Pleasure/Pain & 62 & 59 & 76.13 & 80 & 66 & 66 & 10.13 & 31.60 \\
\hline Ties to Land & 53 & 2 & 2 & 42 & 3 & 3.79 & -1.79 & -8.35 \\
\hline
\end{tabular}

Standardized negative $=$ Severe or Moderate Group total utterances/Severe or Moderate Group total utterances*Severe or Moderate Group negative utterances. Standardized difference in counts $=($ Severe Group total goals and values+Moderate Group total goals and values $) /(\text { Severe Group total theme } \mathrm{X}+\mathrm{Moderate} \text { Group total theme } \mathrm{X})^{*}$ Difference in counts. 
Frequency analysis comparing index and control groups confirmed adverse effects of childhood epilepsy on all dimensions of family well-being except for ties to land. These results also validated the findings of qualitative data comparisons indicating robust effects of childhood epilepsy on family goals and values, daily routines, and social relationships. Comparisons between Sever and Moderate Epilepsy Groups additionally suggested an association between higher seizure frequency and more negative family well-being, with frequency analysis demonstrating especially strong associations of seizure frequency on family resources and goals and values.

Associations between seizure frequency and low wellbeing are consistent with past research on the impact of seizure symptoms on quality of life and highlight the importance of active efforts to control seizures $[17,38,56$, 57]. By demonstrating specific family-level effects of pediatric epilepsy and clarifying how these dimensions are affected, this study documents the value of the ethnographic approach to inform health programs about the effects of pediatric disease.

Negative impact on the siblings in over half of the index families and in all Severe Epilepsy Group families, suggests that siblings of children with seizures in this cultural context, especially those experiencing more frequent seizures, are adversely affected. Existing research in Western cultural contexts $[6,9,58-60]$ has shown that negative impacts reported by siblings result indirectly from the psychological and emotional effects of the child's condition on mothers. Siblings of children in the Kenyan families appeared to experience more direct adverse effects due to unequal distribution of financial resources and disruptions in and limitations on daily activities. These sources of sibling burden are not documented in the existing research. This suggests that an understanding of cultural in terms of family dynamics, structures, and roles is useful in explaining cross cultural differences in the ways that families experience chronic pediatric illness.

In view of the small sample size and requirement that index children have persistent seizures, the findings may not generalize to a broader population of children with epilepsy. A further limitation is the possibility that the index families may have differed from control families in ways other than having to care for a child with epilepsy. Despite efforts to match the groups on background factors, the index families may have functioned less well for other reasons. Furthermore, two of the eight index families included family members other than the index child who experienced seizures, which may have contributed to negative family well-being. A limitation related to frequency analysis is that, as participants in a study of pediatric epilepsy, index family members may have been biased towards speaking negatively about their lives and well-being. Observations and informal discussions were used to help insure that coding of interview material reflected actual family perceptions. Moreover, the differences in family well-being between Severe and Moderate Epilepsy Groups, which were not subject to this bias, were similar to those between the index and control families.

It is also important to acknowledge that results from the group and sub-group comparisons are not evidence for causal relationship between the presence or severity of pediatric epilepsy and family well-being. Specifically, these data do not permit one to determine if the presence and severity of epilepsy has adverse effects on families, or if families with low well-being are more likely to have children with uncontrolled seizures. A bidirectional relationship of seizures and family well-being is also tenable. Studies examining changes in family well-being related to the onset of childhood seizures or to variations over time in seizure severity would strengthen the evidence for a causal link between the child's condition and family well-being.

\section{CONCLUSIONS}

Further research is required to validate these results. Survey research conducted with a larger population of index and control families in Kilifi would be useful in investigating the accuracy and generalizability of the seven dimensions of well-being identified in this study. Exploring family wellbeing in another cultural context using similar methods to those employed here would also be useful in determining if the findings generalize across cultures. More specifically, research using a similar theoretical and methodological approach conducted in a Western context would enable comparisons and test the efficacy of cross-cultural applications of epilepsy intervention models primarily based on research with Western populations. Without comparative research conducted in Western contexts, it is difficult to draw comparisons in the dimensions of well-being described here. It is therefore difficult to see how the findings described here may or may not be applicable in the West or how the Western-based literature on families with children with epilepsy may or may not apply in Non-Western contexts. Similarly, research on other chronic childhood conditions, such as diabetes or asthma, would illuminate the distinct and shared effects of different illnesses on family well-being. These other chronic conditions may place similar demands on caregivers to administer on-going medication regimens, or to take action to avoid symptom exacerbation. However, these conditions may be associated with different cultural perceptions of causation compared to epilepsy and may be less subject to stigma.

Future research is also needed to examine the effectiveness of intervention programs to alleviate the negative family consequences documented in this study. If adverse effects on family well-being can be reduced, families may be better able to cope with their child's needs. The impact of epilepsy on cultural goals and values could be addressed through improvements of doctor/patient dynamics and physician communication strategies. Improving these interactions may assist caregivers in learning about the effectiveness of biomedical anti-epilepsy drugs. Adherence to anti-epilepsy drugs can reduce or eliminate seizures and would allow children to participate more fully in normal household activities, attend school, and avoid stigmatization, thereby improving the daily functioning of the family and facilitating a more positive view of family goals and values. Improved seizure management would also reduce the negative impact of seizure frequency on the quality and predictability of family life. Better management, at least in Kilifi, may also require collaboration with traditional healers [18]. Traditional healers are well accepted in Kilifi and their treatments are culturally meaningful. Encouraging an open 
system of referral between traditional healers and biomedical health workers would increase the number of individuals receiving and adhering to biomedical treatments. Designing a series of public community testimonials featuring individuals with well-managed epilepsy who go to school, have jobs, live normal lives, and achieve cultural goals would also be effective. Reducing the drain on family resources via a transportation voucher system, subsidization of anti-epilepsy drugs, and improved supply of drugs and training of individuals at rural health centers would be other ways to enhance family well-being.

Application of the ethnographic, person-centered approach sheds light on what it means to be "well," and how families are affected by caring for children with a chronic health condition. This knowledge can be used, in turn, to develop efficient assessments of family well-being and provide interventions targeted to specific family needs. Because families assume the major burden of care for these children, helping the family is be a powerful means for improving disease management.

\section{ACKNOWLEDGEMENTS}

We wish to acknowledge support from the Kenya Medical Research Institute, the National Science Foundation, and the Institute of International Education. We also wish to acknowledge Thomas Weisner for his guidance on research design, data analysis, and his comments on drafts of the paper. Finally, we would like to acknowledge Carolina Izquierdo for her comments on drafts and her guidance on the theoretical underpinnings of the research. Charles Newton is funded by the Wellcome Trust, United Kingdom.

\section{REFERENCES}

[1] Austin JK, MacLeod J, Dunn DW, Shen J, Perkins SM. Measuring stigma in children with epilepsy and their parents: instrument development and testing. Epilepsy Behav 2004; 5(4): 472-82.

[2] Hoare P. Psychiatric disturbance in the families of epileptic children. Dev Med Child Neurol 1984; 26(1): 14-9.

[3] Kleinman A, Wang WZ, Li SC, et al. The social course of epilepsy: chronic illness as social experience in interior China. Soc Sci Med 1995; 40(10): 1319-30.

[4] Streisand R, Swift E, Wickmark T, Chen R, Holmes CS. Pediatric parenting stress among parents of children with type 1 diabetes: the role of self-efficacy, responsibility, and fear. J Pediatr Psychol 2005; 30(6): 513-21.

[5] Ziegler R. Epilepsy: individual illness, human predicament and family dilemma. Fam Relat 1982; 31(3): 435-44.

[6] Ellis N, Upton D, Thompson P. Epilepsy and the family: a review of current literature. Seizure 2000; 9(1): 22-30.

[7] Austin JK, Caplan P. Behavioral and psychiatric comorbities in pediatric epilepsy: toward an integrative model. Epilepsia 2007; 48(9): 1639-51.

[8] Pianta RC, Lothman DJ. Predicting behavior problems in children with epilepsy: child factors, disease factors, family stress, and child-mother interaction. Child Dev 1994; 65(5): 1415-28.

[9] Rodenburg R, Meijer AM, Dekovic M, Aldenkamp AP. Family factors and psychopathology in children with epilepsy: a literature review. Epilepsy Behav 2005; 6(4): 488-503.

[10] Rodenburg R, Meijer AM, Dekovic M, Aldenkamp AP. Family predictors of psychopathology in children with epilepsy. Epilepsia 2006; 47(3): 601-14.

[11] Sbarra DA, Rimm-Kaufman SE, Pianta RC. The behavioral and emotional correlates of epilepsy in adolescence: A 7-year follow-up study. Epilepsy Behav 2002; 3(4): 358-67.

[12] Weisner TS. Ecocultural understanding of children's developmental pathways. Hum Develop [Well-being, children, development] 2002; 45: 275-81.
[13] Janzen J. The quest for therapy in lower Zaire. Berkeley and Los Angeles: University of California Press 1978.

[14] Janzen JM. The comparative study of medical systems as changing social systems. Soc Sci Med 1978; 12(2B): 121-33.

[15] Chavunduka GL. Traditional healers and the Shona patient. Gwelo: Mambo Press 1978.

[16] Austin JK, Dunn DW, Johnson CS, Perkins SM. Behavioral issues involving children and adolescents with epilepsy and the impact of their families: recent research data. Epilepsy Behav 2004; 5(Suppl 3): S33-41.

[17] Camfield C, Breau L, Camfield P. Impact of pediatric epilepsy on the family: a new scale for clinical and research use. Epilepsia 2001; 42(1): 104-12

[18] Kendall-Taylor N. Treatment seeking for a chronic disorder: how families in Coastal Kenya make epilepsy treatment decisions. Hum Org 2009; 68(2): 141-53.

[19] Mathews G, Izquierdo C. Pursuits of happiness: well-being in anthropological perspective. New York: Berghahn Books 2008

[20] Ryan G, Bernard HR. Data Management and Analysis Methods. In: Denzin N, Lincoln Y, Eds. Handbook of Qualitative Research. Thousand Oaks: Sage Publications Inc. 2000.

[21] Ryan G, Weisner T. Analyzing words in brief description: fathers and mothers describe their children. Cult Anthropol Methods 1996; 8(3): 13-5.

[22] Adelson N. Being alive well: health and the politics of Cree wellbeing. Toronto: Toronto University Press 2000.

[23] Brim OG, Ryff CD, Kessler RC. How healthy are we?: a national study of well-being at midlife. Chicago: University of Chicago Press 2004.

[24] Clark S. Pleasure experienced: well-being and the Japanese bath. In: Mathews G, Izquierdo C, Eds. Pursuits of happiness: well-being in anthropological perspective. New York: Berghahn Books 2008.

[25] Colby B. Well-being: a theoretical program. Am Anthropol 1987; 89(4): 879-95.

[26] Izquierdo C. When "health" is not enough: societal, individual and biomedical assessments of well-being among the Matsigenka of the Peruvian Amazon. Soc Sci Med 2005; 61(4): 767-83.

[27] Parkin DJ. Sacred void: spatial images of work and ritual among the giriama of Kenya. Cambridge England: Cambridge University Press 1991.

[28] Centre for Geographical Medicine (Coast) KMRI, Kilifi, Kenya, Census Division. Religious Identification: Centre for Geographical Medicine (Coast), Kenya Medical Research Institute, Kilifi, Kenya 2005.

[29] Commission on Epidemiology and Prognosis ILAE. Guidelines for epidemiological studies on epilepsy. Epilepsia 1993; 34: 592-696.

[30] International League Against Epilepsy. Commission Report: The epidemiology of the epilepsies future directions. Epilepsia 1997; 38: 614-8.

[31] El Sharkawy G, Newton C, Hartley S. Attitudes and practices of families and health care personnel toward children with epilepsy in Kilifi, Kenya. Epilepsy Behav 2006; 8(1): 201-12.

[32] Mung'ala-Odera V, White S, Meehan R, et al. Prevalence, incidence and risk factors for epilepsy in older children in rural Kenya. Seizure 2008; 17(5): 396-404.

[33] Shorvon SD. Epidemiology, classification, natural history, and genetics of epilepsy. Lancet 1990; 14; 336(8707): 93-6.

[34] Diop AG, de Boer HM, Mandlhate C, Prilipko L, Meinardi H. The global campaign against epilepsy in Africa. Acta Trop 2003; 87(1) 149-59.

[35] Jilek-Aall LaJ, W. The influence of culture on epilepsy in East Africa. Curare 1993; 16(3): 141-4

[36] Carter J. Epilepsy and developmental impairments following severe malaria in Kenyan children: a study of their prevalence, relationships, clues to pathogenesis and service requirements. $\mathrm{PhD}$ dissertation from University of London 2002.

[37] Edwards T, Scott GA, Munyoko G, et al. Active convulsive epilepsy in a rural district of Kenya: a study of prevalence and possible risk factors. Epilepsy Behav 2008; 8(1): 50-6.

[38] Birbeck GL, Hays RD, Cui X, Vickrey BG. Seizure reduction and quality of life improvements in people with epilepsy. Epilepsia 2002; 43(5): 535-8.

[39] French JA, Kanner AM, Bautista J, Abou-Khalil B, Browne T, Harden CL. Efficacy and tolerability of the new antiepileptic drugs II: treatment of refractory epilepsy: report of the TTA and QSS 
subcommittees of the American academy of neurology and the American epilepsy society. Epilepsia 2004; 45: 410-23.

[40] Levy R, Hollan D. Person-centered interviewing and observation in anthropology. In: Bernard HR, Ed. Handbook of Methods in Cultural Anthropology. Walnut Creek: Alta Mira Press 1998.

[41] Strauss AL. Qualitative analysis for social scientists. New York: Cambridge University Press 1987.

[42] Glaser BG, Strauss AL. The discovery of grounded theory; strategies for qualitative research. Chicago: Aldine Publishers Co. 1967.

[43] Strauss AL, Corbin J. Basics of qualitative research: Grounded theory procedures and techniques. Newbury Park, CA: Sage Publications 1990.

[44] Weber R. Basic content analysis. $2^{\text {nd }}$ ed. Newbury Park, CA: Sage Publications 1990 .

[45] Krippendorff K. Content analysis: An introduction to its methodology. Beverly Hills: Sage Publications 1980.

[46] Altheide D. Ethnographic content analysis. Qual Sociol 1987; 10(1): $65-77$.

[47] Jankowiak W. Well-being, cultural pathology, and personal rejuvenation. In: Mathews $\mathrm{G}$, Izquierdo $\mathrm{C}$, Eds. Pursuits of happiness: well-being in anthropological perspective. New York: Berghahn Books 2008.

[48] Izquierdo C. Well-being among the matsigenka of the peruvian amazon: health, missions, oil, and "progress". In: Mathews G, Izquierdo C, Eds. Pursuits of happiness: well-being in anthropological perspective. New York: Berghahn Books 2008.

[49] Gronseth AS. In search of community: a quest for well-being among Tamil refugees in Northern Norway. Med Anthropol Q 2001; 15(4): 493-514
[50] Edgerton RB. Sick societies: challenging the myth of primitive harmony. New York, Toronto: Free Press; Maxwell Macmillan Canada; Maxwell Macmillan International; 1992.

[51] Bishop M, Allen CA. The impact of epilepsy on quality of life: a qualitative analysis. Epilepsy Behav 2003; 4(3): 226-33.

[52] Devinsky O. Clinical uses of the quality-of-life in epilepsy inventory. Epilepsia 1993; 34(Suppl 4): S39-44.

[53] Beverly E, Whittemore R. Mandinka children and the geography of well-being. Ethos 1993; 21(3): 235-72.

[54] McGregor D, Morelli T, Matuoka J, Minerbi L. An ecological model of wellbeing. In: Becker HA, Vanclay F, Eds. The international handbook of social impact assessment : conceptual and methodological advances. Cheltenham, UK ; Northampton, MA: Edward Elgar 2003; Vol. xxii: p. 326.

[55] Wilcox RR. Fundamentals of modern statistical methods. New York: Springer-Verlag 2001

[56] Baker GA, Jacoby A, Buck D, Stalgis C, Monnet D. Quality of life of people with epilepsy: a European study. Epilepsia 1997; 38(3): 353-62.

[57] Berto P. Quality of life in patients with epilepsy and impact of treatments. Pharmacoeconomics 2002; 20(15): 1039-59.

[58] Hoare P, Kerley S. Psycholsocial adjustment of children with chronic epilepsy and their families. Dev Med Child Neurol 1991; 33: $210-5$.

[59] Hodes M, Garralda ME, Rose G, Schwartz R. Maternal expressed emotion and adjustment in children with epilepsy. J Child Psychol Psychiatry 1999; 40(7): 1083-93.

[60] Long CG, Moore JR. Parental expectations for their epileptic children. J Child Psychol Psychiatry 1979; 20(4): 299-312.

(C) Kendall-Taylor et al.; Licensee Bentham Open.

This is an open access article licensed under the terms of the Creative Commons Attribution Non-Commercial License (http://creativecommons.org/licenses/by$\mathrm{nc} / 3.0 /$ ) which permits unrestricted, non-commercial use, distribution and reproduction in any medium, provided the work is properly cited. 